\title{
Epidemic insurance broker
}

\section{Following the launch of an initiative at the World Economic Forum to prevent future epidemics, we spoke to the scientist at the helm of this coalition, John-Arne Røttingen, who answered our questions on epidemic preparedness, moving from academia to public health policy, evidence-based decision-making, and more.}

Thank you for talking to us, especially at what must be a very busy time for you. For unfamiliar readers, what is your background in research and public health?

I trained as a physician, got involved in research in cellular physiology and did a de facto $\mathrm{MD}-\mathrm{PhD}$ programme. I was also engaged in population and global health and got the opportunity to train and work on infectious disease epidemiology and modelling at Oxford University. This brought me gradually into health policy and services research and global public health, before I returned to infectious diseases in my role as head of infection control at the Norwegian Institute of Public Health.

What made you decide to get involved in public health policy? How would you compare it to academia?

My main motivation for transferring from basic research to health services and public health research was an ambition to see the impact of my work. When you train as a physician, this becomes an ingrained trait. Working in governmental agencies such as the Norwegian Knowledge Centre for Health Services and the Norwegian Institute of Public Health has been a great combination of informing policy and practice while doing research and applied public health work.

You were recently announced as the interim chief executive officer for the Coalition of Epidemic Preparedness Innovations (CEPI). Please tell us what CEPI is. What are its goals?

CEPI is an alliance between governments, industry, philanthropy, intergovernmental institutions and civil society. Its mission is to develop new vaccines to prevent epidemics. It was founded by the Norwegian government, the Indian government's Department of Biotechnology, the Wellcome Trust, the Bill and Melinda Gates Foundation and the World Economic Forum. Other partners include pharmaceutical corporations and non-governmental organizations.

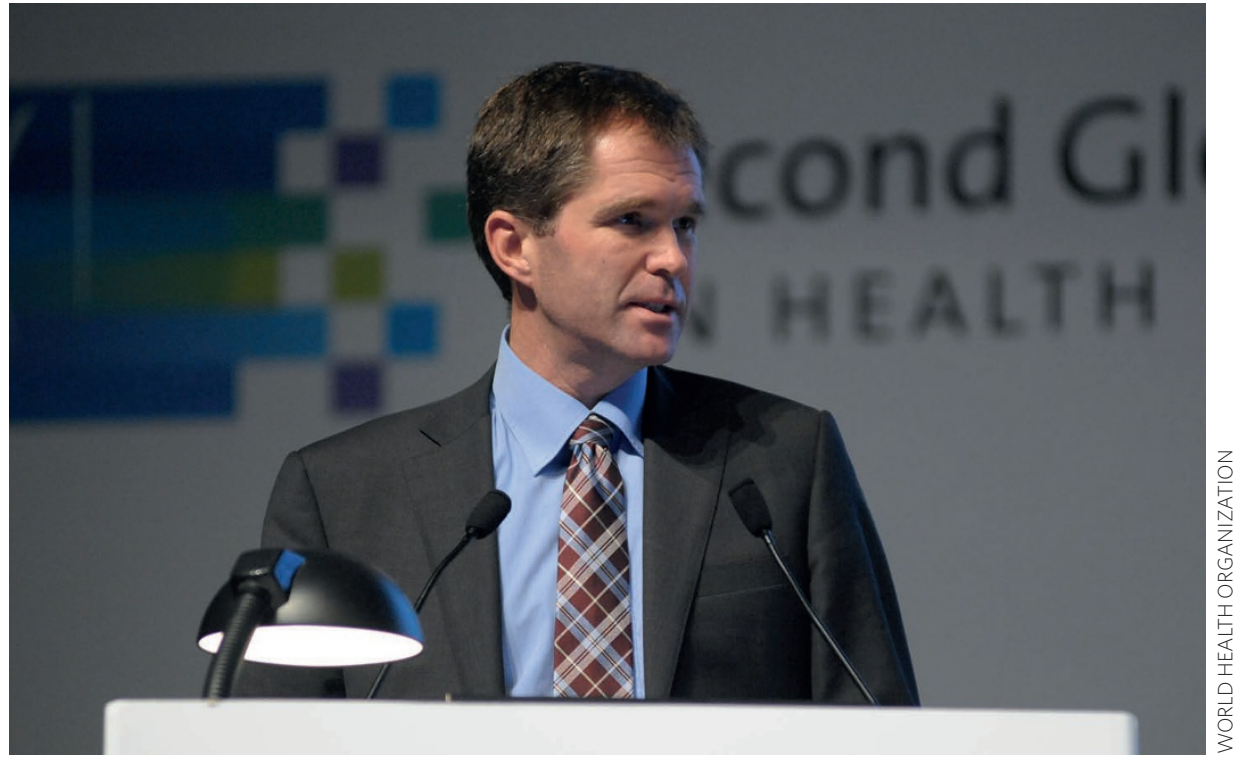

How is CEPI funded, and how will it operate?

CEPI has an initial investment of

US\$540 million from the governments of Germany, Japan and Norway, the Bill and Melinda Gates Foundation and the Wellcome Trust. The European Commission will contribute to CEPI's objectives and plans to co-fund actions with CEPI, such as through the Innovative Medicines Initiative. The government of India is currently finalizing the level of a significant funding commitment.

CEPI will co-ordinate funding and stimulate $\mathrm{R} \& \mathrm{D}$ for vaccines against emerging infectious diseases until they are ready for phase III clinical trials. They can then be deployed early in an outbreak, under emergency conditions, to prevent an epidemic.

CEPI will engage with a range of stakeholders outside of its financing scope, from the discovery phase to manufacturing, procurement and regulation - taking an end-to-end perspective even if only investing in clinical development. These stakeholders are represented in all CEPI governing bodies.

By what criteria were the initial viruses to tackle selected from the list of potential pandemic agents?

The starting point was the World Health
Organization (WHO) list of priority pathogens against which medical countermeasures are urgently needed. CEPI's scientific advisory committee chose three diseases - Middle East respiratory syndrome, Nipah virus and Lassa fever using criteria including the disease's public health impact, the risk of an outbreak occurring and the feasibility of vaccine development, and based on current knowledge, tools and pipeline candidates.

These three diseases are all viral zoonoses, with animal reservoirs and/or intermediary hosts. All have high case fatality rates and can spread in outbreaks. Lassa fever is almost an endemic disease in some locations, and can cause subclinical infections. There are promising vaccines in the pipeline for all three.

What needs to happen for CEPI to include vaccines against the 34 other viruses in the Woolhouse watchlist in its development plans?

CEPI will base its work on the priorities set by WHO. WHO will update its threat list annually and we will follow that process closely. CEPI will probably expand to other pathogens, and the next area will be nonZaire Ebola and Marburg vaccines. However, CEPI will need more investments to be able 
to take on more pathogens. We believe it will be important to deliver on prioritised targets rather than spreading out too thinly.

\section{Why will CEPI focus only on vaccine} development, rather than discovery or delivery?

Development of vaccines against epidemic threats is not really happening today, because the market for such vaccines is very limited. This is a hole in the system for preparedness against epidemic threats. Both discovery and vaccine delivery is functioning better than the clinical development of epidemic vaccines from late preclinical stage and up to phase III. One of the lessons learned from the recent Ebola outbreak in West Africa was that we need a new global R\&D mechanism for developing vaccines before the outbreak happens. If the Ebola vaccine had been ready and we had started the phase III trial six months before, we could have saved thousands of lives.

Timely vaccine development can avert global public health emergencies, contain loss of life, and limit the social and economic damage of outbreaks. CEPI aims to advance safe, effective and affordable vaccines to contain outbreaks at the earliest possible stage. It will give us the joint global insurance policy we need, helping the populations most at risk and making us all safer.

Beyond CEPI, how can the logistical challenges in vaccine storage, delivery and deployment to the places most in need be met?

CEPI is not a delivery-oriented organization. We have therefore started discussions with organizations such as Gavi (the Vaccine Alliance) and UNICEF to better understand potential collaboration and handshake mechanisms, whereby they could invest in stockpiling and deployment in emergencies.

To what degree should governments and health organizations prioritize money to prepare for potential future public health emergencies at the expense of tackling existing chronic problems, infectious diseases or otherwise?

This is a challenging question. It is not an either-or, we need to do both. However, in recent years, global health has focused on where we have most cost-effective interventions that can tackle the larger burden of disease. Such criteria do not take into account the epidemic nature of outbreaks, and the need to invest in preparedness and prevention to reduce the impact of potential future threats. The Ebola outbreak unfortunately demonstrated that we need to invest in preparedness, in the same way we invest in fire brigades in our local communities.

What are the key political, economic or sociological challenges in preparing for epidemic threats at the international scale?

The challenge is that outbreaks are sporadic and often occur in countries that have limited resources for developing countermeasures against the diseases, whereas countries with the resources and infrastructure to invest in such research often feel less threatened. This is a false sense of security; in a world with increased mobility, pathogens also travel and cannot easily be stopped at borders.

The recent Ebola epidemic was a serious wake-up call. Several post-Ebola assessment panels reached the same conclusion: the world needs a new system for coordination of R\&D against epidemic threats. CEPI aims to be this new system. In this case, the global community has demonstrated that it can come together and collaborate, in order to prepare against public health emergencies.

To what extent can the actions of one or a few countries pose a threat to global strategy/outcomes?

We live in the same world and all countries depend on each other. The International Health Regulations (IHR), which are our global framework for working together for global health security, is ratified by 196 countries. Through IHR, countries have agreed to build their capacities to detect, assess and report public health events. WHO plays the coordinating role in IHR and has the authority to declare a
Public Health Emergency of International Concern so that the international community can react and assist.

This is a system built on trust, and countries need to detect and report potential threats to public health as early as possible. If these facts are kept secret, outbreaks will develop too far before the international community is alerted and able to assist.

Wealthier countries have the responsibility to contribute to the protection of vulnerable populations far from their own borders. Protecting the vulnerable is protecting everybody, but this fact is often hard to convey to people in more privileged positions.

One important part of countries responsibilities is to collaborate and share health data and samples. However, both data and sample sharing need to be done within a framework where the insights and potential products can also be accessed by those who contribute. We need benefit sharing arrangements, but these cannot become barriers to effective scientific and public health collaboration.

Has evidence-based decision-making in public health policy been affected by recent changes in the political climate? I do not think so. We need more evidence-informed policies and decisions. The outgoing administration in the United States initiated a Commission on Evidence-Based Policymaking with bipartisan support, and it will be important to build on their conclusions when they are issued.

Lastly, what would your advice be for early career scientists considering working in public health and policy? Don't plan. Follow your heart and interests and opportunities will arise. Most scientific topics have a policy and a potential public health aspect to them. Scientists with the right aptitudes have both a right and a responsibility to engage in the policy arena.

INTERVIEW BY NONIA PARIENTE 